\title{
Spectral Representation for Branching Processes on the Real Half Line
}

\author{
By \\ Yukio OGURA*
}

\section{$\S 0$. Introduction}

The spectral theory for the semigroups of Galton-Watson processes (G.W.P.'s) was developed by Karlin and McGregor [4] [5]. On the other hand, a continuous state branching process (C.B.P.), which may be regarded as a continuous version of a G.W.P., was introduced by Jirina [3] and recently discussed by Lamperti [7], S. Watanabe [8], etc. The object of this paper is to obtain the spectral representation theorems for C.B.P.'s similar to those of Karlin and McGregor for G.W.P.'s. These may be of some interest, since there are many C.B.P.'s with discontinuous sample functions and their semigroups are nonsymmetrizable.

In $\S 1$, we obtain a representation (see (1.10)) of a so called $\Psi$-semigroup by means of the stationary measure, under the assumption that the extinction probability is positive. In $\S 2$, we shall prepare several lemmas. The most important one is Lemma 2.4, which asserts that, under Condition A, the representation of a $\Psi$ semigroup in $\S 1$ turns out to be a spectral representation of the transition function. In $\S 3$, the spectral representation theorem for sub- and supercritical C.B.P.'s is given. In this representation, only the discrete spectrum appears which consists of powers of the largest eigenvalue. In $\S 4$, the critical case is considered. In this case, the spectrum is continuous. The asymptotic behavior of the semigroup is also obtained. In $\S 5$, we give a few examples which contain all diffusion C.B.P.'s.

The author wishes to express his thanks to Professor K. Ito

Received November 12, 1969.

Communicated by $\mathrm{K}$. Yosida.

* Department of Applied Science, Faculty of Engineering, Kyushu University. 
and Professor N. Ikeda for their valuable discussions. The author also wishes to express his deep thanks to Professor S. Watanabe for his fruitful suggestions and encouragement.

\section{§. A Representation of a $\Psi$-semigroup}

A C.B.P. is a Markov process $\left(x_{t}, P_{x}\right)$ on the real half line $[0, \infty]$ with $\infty$ as a trap, which satisfies, for every $\lambda>0$,

$$
E_{x}\left(e^{-\lambda x} t\right)=e^{-x \psi_{t}^{(\lambda)}}
$$

for some $\psi_{t}(\lambda)>0$. Then it is easy to see that $\psi_{t}(\lambda)$ has a completely monotone derivative in $\lambda$ for each $t \geq 0$, and satisfies the semigroup property ;

$$
\psi_{t+s}(\lambda)=\psi_{t}\left(\psi_{s}(\lambda)\right), \quad \psi_{0}(\lambda)=\lambda .
$$

Such a $\left\{\psi_{t}(\lambda)\right\}_{t \in[0, \infty)}$ is called a $\Psi$-semigroup ${ }^{1)}$ and it is equivalent to give a C.B.P. and to give a $\Psi$-semigroup. We shall assume that $\psi_{t}(\lambda)$ is differentiable in $t$ throughout this paper. (For this, it is sufficient to assume $\psi_{t}(\lambda)$ is continuous in $t$, cf. [8].) Then $h(\lambda) \equiv$ $\partial \psi_{t}(\lambda) /\left.\partial t\right|_{t=0}$ has the form

$$
h(\lambda)=-a \lambda^{2}+b \lambda+c-\int_{0}^{\infty}\left(e^{-\lambda y}-1+\lambda(y \wedge 1)\right) n(d y),
$$

where $a, b$ and $c$ are real constants with $a \geq 0, c \geq 0$, and $n$ is a nonnegative measure on $(0, \infty)$ with $\int\left(y^{2} \wedge 1\right) n(d y)<\infty$ (see [8]). From (1.2), it is clear that

$$
\frac{\partial}{\partial t} \psi_{t}(\lambda)=h\left(\psi_{t}(\lambda)\right), \quad \psi_{0}(\lambda)=\lambda, \quad \lambda>0 .
$$

Conversely, for a given $h(\lambda)$ with above properties, the solution $\psi_{t}(\lambda)$ of (1.4) defines a $\Psi$-semigroup (cf. [8]).

Now we shall divide C.B.P.'s into three classes as in the case of G.W.P.'s.

1) $\Psi$ is the class of functions with completely monotone derivatives;

$$
\begin{gathered}
\Psi=\left\{\psi(\lambda)=c_{0}+c_{1} \lambda+\int_{0}^{\infty}\left(1-e^{-\lambda u}\right) n(d u) ; c_{0} \geq 0, c_{1} \geq 0,\right. \\
n \text { is a nonnegative measure on }(0, \infty) \text { such that } \\
\left.\qquad \int_{0}^{\infty}(1 \wedge u) n(d u)<\infty\right\} .
\end{gathered}
$$


Definition 1.1. The function $h(\lambda)$ is called supercritical (subcritical, critical) if $h^{\prime}(0)>0$ or $c>0$ (resp. $h^{\prime}(0)<0$ and $c=0$, resp. $h^{\prime}(0)=0$ and $c=0) . A$ C.B.P. is called supercritical (subcritical, critical) if its $h(\lambda)$ is so.

Throughout this paper, we shall assume

$$
\int_{\lambda}^{\infty} \frac{d \tau}{h(\tau)} \text { converges for a large } \lambda \text {. }
$$

Note that (1.5) implies $h(\lambda)<0$ for large $\lambda$, since $h(\lambda)$ is concave. Thus, if $h(\lambda)$ is supercritical, the equation $h(\lambda)=0$ has unique positive solution $\gamma$. When $h(\lambda)$ is not supercritical, we set $\gamma=0$ for convenience. (1.5) is equivalent to a simple probabilitistic condition (cf. Remark 1.1 below).

Lemma 1.1. It holds that

$$
\lim _{t \rightarrow \infty} \psi_{t}(\lambda)=\gamma, \quad \lambda>0 .
$$

Proof. From (1.4), $d \psi_{t}(\lambda) / d t$ is positive (negative) if $0<\psi_{t}(\lambda)<\gamma$ $\left(\gamma<\psi_{t}(\lambda)\right)$, so that $\psi_{t}(\lambda)$ is nondecreasing (nonincreasing) if $0<\lambda<\gamma$ $(\gamma<\lambda)$. Hence the limit $\gamma^{\prime}=\lim _{t \rightarrow \infty} \psi_{t}(\lambda)$ exists, and $\lim _{t \rightarrow \infty} d \psi_{t}(\lambda) / d t=0$. If $\gamma^{\prime} \neq \gamma$, by (1.4) $0=\lim _{t \rightarrow \infty} d \psi_{t}(\lambda) / d t=h\left(\gamma^{\prime}\right) \neq 0$, which is a contradiction.

As the case of a G.W.P., a stationary measure for a C.B.P. on $[0, \infty)$ turns out to be trivial (cf. [2] pp. 23 24). So we shall deal with a stationary measure on $(0, \infty)$.

Definition 1.2. A nonnegative measure $\pi(E)$ on $(0, \infty)$ is called a stationary measure for a C.B.P., if it satisfies

$$
\int_{0+}^{\infty} \pi(d x) P_{t}(x, E)=\pi(E), \quad E \in \mathcal{K}(0, \infty),{ }^{2)}
$$

and $0<\hat{\pi}(\lambda)<\infty$ for sufficiently large $\lambda$, where we set

$$
\hat{\pi}(\lambda)=\int_{0+}^{\infty} e^{-\lambda x} \pi(d x) .
$$

Lemma 1.2. There is a stationary measure, whose Laplace transform is given by

2) $\mathcal{K}(0, \infty)(\mathcal{K}[0, \infty))$ is the class of all Borel measurable sets in $(0, \infty)($ resp. $[0, \infty))$ with compact closures. 


$$
\hat{\pi}(\lambda)=-\int_{\lambda}^{\infty} \frac{d \tau}{h(\tau)}, \quad \lambda>\gamma .
$$

$\hat{\pi}(\lambda)$ satisfies

$$
\hat{\pi}\left(\psi_{t}(\lambda)\right)=\hat{\pi}(\lambda)+t, \quad \lambda>\gamma .
$$

Proof. First, we shall show that $\hat{\pi}(\lambda)$ in (1.8) is completely monotone. By (1. 8), $-\hat{\pi}^{\prime}(\lambda)=(1 / \lambda)(1 / \psi(\lambda))$, where $\psi(\lambda)=-h(\lambda) / \lambda$. Since $1 / \lambda$ is completely monotone, it is enough to show that $\psi(\lambda)$ $(>0)$ has a completely monotone derivative ([1] p. 417). But this follows from the equality

$$
\frac{d^{n}}{d \lambda^{n}} \psi(\lambda)=(-1)^{n+1} \frac{n !}{\lambda^{n+1}} \sum_{\nu=0}^{n} \frac{(-\lambda)^{\nu}}{\nu !} \frac{d^{\nu}}{d \lambda^{\nu}} h(\lambda) .
$$

Next, (1.4) implies $-\int_{\psi_{t}(\lambda)}^{\lambda} \frac{d \tau}{h(\tau)}=t$, that is

$$
\hat{\pi}\left(\psi_{t}(\lambda)\right)=\hat{\pi}(\lambda)+t, \quad \lambda>\gamma .
$$

Since

$$
\hat{\pi}\left(\psi_{t}(\lambda)\right)=\int_{0+}^{\infty} \int_{0+}^{\infty} \pi(d x) P_{t}(x, d y) e^{-\lambda y}+\int_{0+}^{\infty} \pi(d x) P_{t}(x,\{0\}),
$$

and

$$
\int_{0+}^{\infty} \pi(d x) P_{t}(x,\{0\})=\lim _{\lambda \rightarrow \infty} \hat{\pi}\left(\psi_{t}(\lambda)\right)=t
$$

by Lebesgue's convergence theorem, we obtain (1.7) from (1.9).

$$
\text { q.e.d. }
$$

Since $-\infty<h^{\prime}(\gamma) \leq 0, \lim _{\lambda \downarrow \gamma} \hat{\pi}(\lambda)=\infty$ by (1.8). Hence $\hat{\pi}(\lambda)$ maps $(\gamma, \infty)$ onto $(\infty, 0)$ monotonously, and the inverse function $\hat{\theta}(w)$ of $\hat{\pi}(\lambda)$ maps $(0, \infty)$ onto $(\infty, \gamma)$ monotonously. Moreover $\hat{\theta}(w)$ is real analytic on $(0, \infty)$, since $\hat{\pi}(\lambda)$ is completely monotone on $(\gamma, \infty)$ and so real analytic there.

Proposition 1.1. The semigroup $\psi_{t}(\lambda)$ has the representation

$$
\psi_{t}(\lambda)=\hat{\theta}(\hat{\pi}(\lambda)+t), \quad \lambda>\gamma, t \geq 0 .
$$

Hence, we have

$$
P_{x}\left(x_{t}=0\right)>0, \quad t>0, x \in[0, \infty) .
$$

Proof. (1.10) is obvious by (1.9). (1.11) follows from (1.10), 
since $P_{x}\left(x_{t}=0\right)=e^{-x \psi_{t}^{(\infty)}}$ where $\psi_{t}(\infty)=\lim _{\lambda \rightarrow \infty} \psi_{t}(\lambda)$, and $\psi_{t}(\infty)=\hat{\theta}(t)<\infty$.

Remark 1. 1. We have seen that (1.11) follows from (1.5). The converse is also true. Indeed, if (1.11) is true for some $t_{0}>0$, $\psi_{t_{0}}(\infty)<\infty$ and so $\lim _{t \rightarrow \infty} \psi_{t}(\infty)<\infty$ since $\psi_{t}(\infty)$ is decreasing function in t. Thus $\lim _{t \rightarrow \infty} \psi_{t}(\lambda) \leq \lim _{t \rightarrow \infty} \psi_{t}(\infty)<\infty$, which implies that $h(\lambda)$ is negative for large $\lambda$ (cf. Lemma 1.1). Since $\psi_{t_{0}}(\infty)$ is larger than the largest solution of $h(\lambda)=0$, we have

$$
-\int_{\psi_{t}(\infty)}^{\infty} \frac{d \tau}{h(\tau)}=\lim _{\lambda \rightarrow \infty} \int_{\psi_{t}(\lambda)}^{\lambda} \frac{d \tau}{-h(\tau)}=t .
$$

Proposition 1.2. Let $\sigma_{\{0\}}$ be the first hitting time for the state 0 . Then we have

$$
P_{x}\left(\sigma_{\{0\}}<\infty\right)=e^{-\gamma x} .
$$

Proof. Since $\psi_{s}(\infty)<\infty$ for $s>0$, Lemma 1.1 implies

$$
\lim _{t \rightarrow \infty} \psi_{t}(\infty)=\lim _{t \rightarrow \infty} \psi_{t}\left(\psi_{s}(\infty)\right)=\gamma
$$

Hence (1.12) follows from (1.1) and the fact that the state 0 is trap. q.e.d.

Since $\lim _{t \downarrow 0} \psi_{t}(\infty)=\infty,(1.13)$ means that $\psi_{t}(\infty)$ maps $(0, \infty)$ onto $(\gamma, \infty)$ as a function of $t$.

Proposition 1.3. A stationary measure for a C.B.P. is unique up to a constant multiple.

Proof. Let $\pi_{0}(E)$ be a stationary measure. Then for $\lambda>\gamma$ and $t>0$ such that $\hat{\pi}_{0}(\lambda)$ and $\hat{\pi}_{0}\left(\psi_{t}(\infty)\right)$ converge, we have from (1. 7) that

$$
\hat{\pi}_{0}\left(\psi_{t}(\lambda)\right)=\hat{\pi}_{0}(\lambda)+\hat{\pi}_{0}\left(\psi_{t}(\infty)\right) .
$$

Since $\psi_{t}(\infty)$ maps $(0, \infty)$ onto $(\gamma, \infty)$, there exists a positive number $t_{0}$ such that $\hat{\pi}_{0}\left(\psi_{t_{0}}(\infty)\right)$ converges. Setting $\lambda=\psi_{t_{0}}(\infty)$ and $t=t_{0}$ in (1.14), we see that $\hat{\pi}_{0}\left(\psi_{2 t_{0}}(\infty)\right)=\hat{\pi}_{0}\left(\psi_{t_{0}}\left(\psi_{t_{0}}(\infty)\right)\right)=2 \hat{\pi}_{0}\left(\psi_{t_{0}}(\infty)\right)$ converges. Repeating this argument, it is clear that $\hat{\pi}_{0}\left(\psi_{n t_{0}}(\infty)\right)$ converges for each integer $n$. Hence $\hat{\pi}_{0}(\lambda)$ converges for each $\lambda>\gamma$ by means of (1.13), so that (1.14) is valid for $\lambda>\gamma$.

Setting $\lambda=\psi_{s}(\infty)$ in (1. 14), we have $\hat{\pi}_{0}\left(\psi_{t+s}(\infty)\right)=\hat{\pi}_{0}\left(\psi_{s}(\infty)\right)+$ $\hat{\pi}_{0}\left(\psi_{t}(\infty)\right)$. Hence, by the continuity of $\psi_{t}(\infty) \hat{\pi}_{0}\left(\psi_{t}(\infty)\right)=c t$ for some $c>0$. Therefore, by (1.14) 


$$
\hat{\pi}_{0}\left(\psi_{t}(\lambda)\right)=\hat{\pi}_{0}(\lambda)+c t
$$

for some $c>0$. Differentiation of (1.15) at $t=0$ implies $\hat{\pi}_{0}^{\prime}(\lambda) h(\lambda)=c$, whose solution with $\lim _{\lambda \rightarrow \infty} \hat{\pi}_{0}^{\prime}(\lambda)=0$ is unique, and is given by $c \hat{\pi}(\lambda)$. Therefore $\pi_{0}=c \pi$, where $\pi$ is the stationary measure of Lemma 1.2.

q.e.d.

\section{§. Some Lemmas}

In this section, we prepare some lemmas which will be used for the spectral representation theorem later.

Lemma 2. 1. Let $u \in[0, \infty)$. Then we have a signed measure $\xi_{u}(d x)$ on $\mathcal{K}[0, \infty)$ satisfying

$$
\begin{array}{ll}
\int_{0}^{\infty} e^{-\lambda x} \xi_{u}(d x)=e^{-u \hat{u}(\lambda)}, & \lambda>\gamma, \\
\int_{0}^{\infty} e^{-\lambda x}\left|\xi_{u}\right|(d x) \leq e^{u \hat{\pi}(\lambda)}, & \lambda>\gamma,
\end{array}
$$

where $\left|\xi_{u}\right|(E)$ is the total variation of $\xi_{u}$ on $E$.

Proof. Since $u \hat{\pi}(\lambda)$ is completely monotone in $\lambda>\gamma$, and the functions $\cosh x$ and $\sinh x$ are absolutely monotone, the functions $\cosh u \hat{\pi}(\lambda)$ and $\sinh u \hat{\pi}(\lambda)$ are completely monotone on $\lambda>\gamma$. Hence we have nonnegative measures $\xi_{u}^{ \pm}(E)$ on $\mathcal{K}[0, \infty)$ satisfying

$$
\int_{0}^{\infty} e^{-\lambda x} \xi_{u}^{ \pm}(d x)=\frac{1}{2}\left(e^{u \hat{\imath}(\lambda)} \pm e^{-u \hat{\pi}(\lambda)}\right), \quad \lambda>\gamma .
$$

Setting $\xi_{u}(E)=\xi_{u}^{+}(E)-\xi_{u}^{-}(E)$, we have (2.1) and (2.2).

Lemma 2.2. $\xi_{u}(E)$ is a eigenmeasure of $P_{t}(x, E)$, in the sense that

$$
\int_{0}^{\infty} \xi_{u}(d x) P_{t}(x, E)=e^{-u t} \xi_{u}(E), \quad E \in \mathcal{K}[0, \infty) .
$$

Proof. By (1.9) and (2.1),

$$
e^{-u \hat{\imath}\left(\psi_{t}(\lambda)\right)}=\int_{0}^{\infty} e^{-\lambda y} e^{-u t} \xi_{u}(d y), \quad \lambda>\gamma .
$$

By (2.2) and (1.1)

$$
\int_{0}^{\infty}\left|\xi_{u}\right|(d x) \int_{0}^{\infty} P_{t}(x, d y) e^{-\lambda y}=\int_{0}^{\infty}\left|\xi_{u}\right|(d x) e^{-x \psi} t^{(\lambda)} \leq e^{u \hat{\pi}\left(\psi_{t}(\lambda)\right)}<\infty .
$$


Hence by Fubini's theorem, we have

$$
e^{-u \hat{\pi}\left(\psi_{t}(\lambda)\right)}=\int_{0}^{\infty} e^{-\lambda y} \int_{0}^{\infty} \xi_{u}(d x) P_{t}(x, d y), \quad \lambda>\gamma .
$$

(2.4) (2.5) and the uniqueness theorem of Laplace transforms ([9] p. 80) implies (2.3).

The same arguments for the function $\hat{\theta}(w)$ is not always clear. So we shall proceed with some assumptions for a while.

Condition A. For each $x \in[0, \infty)$, there is a signed measure $\phi_{E}(x)$ on $[0, \infty)$, satisfying

$$
\begin{array}{ll}
\int_{0}^{\infty} e^{-w u} \phi_{d u}(x)=e^{-x \hat{\theta}(w)}, & w>t_{0}, \\
\int_{0}^{\infty} e^{-w u}|\phi|_{d u}(x)<\infty, & w>t_{0},
\end{array}
$$

for some $t_{0} \geq 0$, where $|\phi|_{E}(x)$ is the total variation of $\phi .(x)$ on $E$.

We shall set $\Phi_{w}(x)=\int e^{-w u}|\phi|_{d u}(x)$. Then the second assumption is :

Condition B. Condition $A$ is satisfied. Furthermore, for each $t>0$,

$$
\int_{0}^{\infty} P_{t}(x, d y) \Phi_{w}(y)<\infty, \quad w>t_{1},
$$

for some $t_{1} \geq t_{0}$, which may depend on $t$.

Lemma 2. 3. If $\hat{\theta}(w)$ satisfies Condition $B, \phi_{E}(x)$ is an eigenfunction of $P_{t}(x, d y)$, in the sense that

$$
\int_{0}^{\infty} P_{t}(x, d y) \phi_{E}(y)=\int_{E} e^{-u t} \phi_{d u}(x), \quad E \in \mathcal{K}[0, \infty) .
$$

Proof. Since the representation (1.10) of $\psi_{t}(\lambda)$ implies $\psi_{t}(\hat{\theta}(w))$ $=\hat{\theta}(w+t)$,

$$
\begin{aligned}
\int_{0}^{\infty} P_{t}(x, d y) \int_{0}^{\infty} e^{-u w} \phi_{d u}(y) & =e^{-x \hat{\theta}(w+t)} \\
& =\int_{0}^{\infty} e^{-u w} e^{-u t} \phi_{d u}(x), \quad w>t_{0} .
\end{aligned}
$$

But by Condition $\mathrm{B}$

$$
\int_{0}^{\infty} P_{t}(x, d y) \int_{0}^{\infty} e^{-u w}|\phi|_{d u}(y)=\int_{0}^{\infty} P_{t}(x, d y) \Phi_{w}(y)<\infty, \quad w>t_{1} .
$$


Hence, Fubini's theorem and the uniqueness theorem of Laplace transforms imply (2.9).

Lemma 2.4. If $\hat{\theta}(w)$ satisfies condition $A, P_{t}(x, E)$ admits a spectral representation

$$
\begin{aligned}
P_{t}(x, E)=\int_{0}^{\infty} \phi_{d u}(x) e^{-u t} \xi_{u}(E), & t>t_{0}, x \in[0, \infty), \\
& E \in \mathcal{K}[0, \infty) .
\end{aligned}
$$

Proof. Fix a time $t>t_{0}$. Then there exists a positive number $L$ such that $t-\hat{\pi}(\lambda)>t_{0}$ for all $\lambda>L$. Hence

$$
\int_{0}^{\infty}|\phi|_{d u}(x) e^{-u t} \int_{0}^{\infty}\left|\xi_{u}\right|(d y) \leq \int_{0}^{\infty}|\phi|_{d u}(x) e^{-u(t-\hat{\pi}(\lambda))}<\infty, \quad \lambda>L .
$$

Thus the integral in (2.10) converges absolutely. Moreover, we can use the Fubini's theorem, so that (1.10) implies

$$
\begin{aligned}
\int_{0}^{\infty} e^{-\lambda y} P_{t}(x, d y) & =e^{-x \hat{\theta}(\hat{\pi}(\lambda)+t)} \\
& =\int_{0}^{\infty} e^{-\lambda y} \int_{0}^{\infty} \phi_{d u}(x) e^{-u t} \xi_{u}(d y), \quad \lambda>L .
\end{aligned}
$$

By the uniqueness theorem of Laplace transform, we have (2.10).

\section{§3. A Spectral Representation with Discrete Spectrum}

In this section, we shall deal with a C.B.P., whose $h(\lambda)$ satisfies

$$
h^{\prime}(\gamma)<0 \text {, and } h(\lambda) \text { is analytic at } \gamma \text {. }
$$

When a C.B.P. is supercritical, (3.1) is automatically satisfied : when it is subcritical, since $h^{\prime}(\gamma)=h^{\prime}(0)<0$ holds automatically, the only assumption is that $h(\lambda)$ is analytic in some neighbourhood of $\lambda=0$.

Lemma 3.1. Let $\mu=-h^{\prime}(\gamma)$. Then (3.1) implies that $A(\lambda)$ $\equiv e^{-\mu \hat{\pi}(\lambda)}$ is analytic at $\gamma$ and $A^{\prime}(\gamma)>0$.

Proof. By (3.1),

$$
\frac{1}{h(\lambda)}=-\frac{1}{\mu(\lambda-\gamma)}\left(1+g_{1}(\lambda-\gamma)\right)
$$

where $g_{1}(x)$ is analytic at 0 and $g_{1}(0)=0$. Therefore, if $\lambda$ is sufficiently near to $\gamma$, 


$$
\begin{aligned}
-\mu \hat{\pi}(\lambda) & =-\int_{\lambda}^{\gamma+\varepsilon} \frac{d \tau}{\tau-\gamma}-\int_{\lambda}^{\gamma+\varepsilon} \frac{g_{1}(\tau-\gamma)}{\tau-\gamma} d \tau+\mu \int_{\gamma+\varepsilon}^{\infty} \frac{d \tau}{h(\tau)} \\
& =\log (\lambda-\gamma)+g(\lambda-\gamma)
\end{aligned}
$$

where $g(x)$ is analytic at 0 and $g^{\prime}(x)=g_{1}(x) / x$. Hence $A(\lambda)=$ $(\lambda-\gamma) e^{g(\lambda-\gamma)}$ is analytic at $\gamma$ and $A^{\prime}(\gamma)=e^{g(0)}>0$.

Since $A(\gamma)=0$, Lemma 3.1 implies that the inverse function $B(v)$ of $A(\lambda)$ is analytic on a neighbourhood $V(0)$ of 0 , and $B(0)=\gamma$. Defining functions $\left\{\phi_{k \mu}(x) ; k=0,1,2, \cdots\right\}$ by

$$
\sum_{k=0}^{\infty} \phi_{k \mu}(x) v^{k}=e^{-x_{B(v)}}, \quad v \in V(0),
$$

we have :

Theorem 3.1. Let (3.1) be satisfied. Then, 1) $\phi_{k \mu}(x)$ is an eigenfunction of $P_{t}(x, E)$ corresponding to the eigenvalue $e^{-k \mu t}$, 2) $P_{t}(x, E)$ admits a spectral representation

$$
\begin{aligned}
P_{t}(x, E)=\sum_{k=0}^{\infty} \phi_{k \mu}(x) e^{-k \mu t} \xi_{k \mu}(E), \quad & t>t_{0}, x \in[0, \infty), \\
& E \in \mathcal{K}[0, \infty) .
\end{aligned}
$$

Proof. First we shall show 2) by checking Condition $A$ in $\S 2$. Since $\pi(\lambda)=-\log A(\lambda) / \mu$ by the definition, $\hat{\theta}(w)=B\left(e^{-\mu w}\right)$ for $w>t_{0}$, where $t_{0}$ is a positive number such that $e^{-\mu t_{0}} \in V(0)$. Hence (2.6) follows, in which $\phi_{E}(x)$ is given by

$$
\phi_{E}(x)=\sum_{k=0}^{\infty} \phi_{k \mu}(x) \delta_{\mu_{k}}(E) .
$$

(2. 7) is obvious, since

$$
\int_{0}^{\infty} e^{-u w}|\phi|_{d u}(x)=\sum_{k}\left|\phi_{k \mu}(x)\right| e^{-k \mu w}<\infty, \quad w>t_{0} .
$$

Next we shall show 1) by checking Condition $B$ in $\S 2$. Fix a positive time $t$. Then the ordinary successive approximation method shows that $\psi_{t}(\lambda)$ is regular on a neighbourhood $U(\gamma)$ of $\gamma$ by means of (3.1) (cf. [8]). Hence $e^{-x \psi_{t}^{(\lambda)}}$ is analytic on $U(\gamma)$, and therefore

$$
\int_{0}^{\infty} P_{t}(x, d y) e^{-\lambda y}=e^{-x \psi_{t}(\lambda)}<\infty, \quad \lambda \in U(\gamma) .
$$

Since $B(v)$ is analytic at 0 and $B(0)=\gamma, B(v)$ has the Taylor's expansion $B(v)=\gamma+\sum_{j \geq 1} b_{j} v^{j}$. So 


$$
\begin{aligned}
\int_{0}^{\infty} e^{-u w}|\phi|_{d u}(x) & =\sum_{k}\left|\phi_{k \mu}(x)\right| e^{-k \mu w} \\
& \leq e^{-\gamma x} \exp \left(x \sum_{j \geq 1}\left|b_{j}\right| e^{-j \mu w}\right), \quad w>t_{0} .
\end{aligned}
$$

Hence, taking $t_{1}$ so large that $\gamma-\sum_{j \geq 1}\left|b_{j}\right| e^{-j \mu t}{ }_{1} \in U(\gamma)$, we have (2.8) by (3.5).

Remark 3.1. If $A(\lambda)$ is defined and analytic until its value goes down to $-1, t_{0}$ in (3.3) may be taken to be 0 . Indeed, if so, $B(v)$ is analytic on $(-1,1)$, (note that $\lim _{\lambda \rightarrow \infty} A(\lambda)=1$ ), and $\hat{\theta}(w)=B\left(e^{-\mu w}\right)$ is analytic on $w>0$.

Especially; if $h(\lambda)$ is analytic on $\lambda>\gamma_{0}$, where $\gamma_{0}$ is the other solution of $h(\lambda)=0$ (if it exists), ${ }^{3}$ then $A(\lambda)$ satisfies the condition: Indeed, in this case $A(\lambda)$ is analytic on $\lambda>\gamma_{0}$ and $\lim _{\lambda \downarrow \gamma_{0}} A(\lambda)=-\infty$.

Remark 3.2. $\phi_{k \mu}(x)=e^{-\gamma x} \times($ a polynomial in $x$ with the degree $k$ ). This follows immediately from

$$
\sum_{k} \phi_{k \mu}(x) v^{k}=e^{-\gamma x} \exp \left(-x \sum_{j \geq 1} b_{j} v^{j}\right)
$$

and the fact that $b_{1}=B^{\prime}(0)=1 / A^{\prime}(\gamma) \neq 0$.

Note that in our case, the representation (1.10) of $\psi_{t}(\lambda)$ turns out to be

$$
\psi_{t}(\lambda)=B\left(e^{-\mu t} A(\lambda)\right) \text {, for large } t .
$$

Proposition 3.1. If (3.1) is valid, then

$$
\psi_{t}(\lambda)=\gamma+e^{-\mu t} \frac{A(\lambda)}{A^{\prime}(\gamma)}+0\left(e^{-2 \mu t}\right), \quad \text { as } t \rightarrow \infty,
$$

or equivalently

$$
E_{x}\left(e^{-\lambda x} t\right)=e^{-\gamma x}-x e^{-\mu t} \frac{A(\lambda) e^{-\gamma x}}{A^{\prime}(\gamma)}+0\left(e^{-2 \mu t}\right), \quad \text { as } \quad t \rightarrow \infty,
$$

where $0\left(e^{-2 \mu t}\right)$ is uniform in $\lambda \geq \gamma-\varepsilon$ for some $\varepsilon>0$. Furthermore it holds that

$$
P_{x}\left(\sigma_{\{0\}} \leq t\right)=e^{-\gamma x}-x e^{-\mu t} \frac{e^{-\gamma x}}{A^{\prime}(\gamma)}+0\left(e^{-2 \mu t}\right), \quad \text { as } \quad t \rightarrow \infty
$$

Proof. Since $B(v)$ is analytic at 0 ,

3) This condition is always satisfied, if $h(\lambda)$ is supercritical with $c=0$. 


$$
B(v)=\gamma+B^{\prime}(0) v+0\left(v^{2}\right), \quad v \rightarrow 0 .
$$

Hence (3. 7) follows from (3.6). (3.8) follows from (1.1) and (3.7). Since $\lim _{\lambda \rightarrow \infty} A(\lambda)=1$, and $B\left(e^{-\mu t} v\right)$ is continuous in $v$ at $v=1$ if $t$ is sufficiently large, we have $\psi_{t}(\infty)=B\left(e^{-\mu_{t}}\right)$ by (3.6). Hence (3.9) follows.

\section{$\S 4$. A Spectral Representation with Continuous Spectrum}

In this section, we shall examine a C.B.P., whose $h(\lambda)$ satisfies

$$
\begin{array}{ll}
h(\lambda)=\lambda^{1+p} g(\lambda), & \text { where } 0<p \leq 1 \text { and } g(\lambda) \text { is } \\
& \text { analytic at } 0 \text { with } g(0) \neq 0 .
\end{array}
$$

Of course (4.1) is satisfied only when $h(\lambda)$ is critical. Since $h(\lambda)$ is concave, $g(0)<0$, and we set $\alpha=-g(0)$ and $\beta=g^{\prime}(0) / 2$. Then if $\lambda$ is sufficiently near 0 ,

$$
h(\lambda)=-\alpha \lambda^{1+p}\left(1-\frac{\beta}{\alpha} \lambda+g_{1}(\lambda)\right),
$$

where $g_{1}(\lambda)$ is regular at 0 with $g_{1}(0)=g_{1}^{\prime}(0)=0$. Hence

$$
\frac{1}{h(\lambda)}=-\frac{1}{\alpha \lambda^{1+p}}-\frac{\beta}{\alpha^{2} \lambda^{p}}+\frac{1}{\lambda^{p}} g_{2}(\lambda)
$$

where $g_{2}(\lambda)$ is regular at 0 with $g_{2}(0)=g_{2}^{\prime}(0)=0$, so that

$$
\hat{\pi}(\lambda)= \begin{cases}\frac{1}{\alpha}\left\{\frac{1}{\lambda}-\frac{\beta}{\alpha} \log \lambda+g_{3}(\lambda)\right\}, & \text { if } p=1, \\ \frac{1}{\alpha p}\left\{\frac{1}{\lambda^{p}}+c+\lambda^{1-p} g_{4}(\lambda)\right\}, & \text { if } 0<p<1,\end{cases}
$$

where $g_{3}(\lambda)$ and $g_{4}(\lambda)$ are regular at 0 and $c$ is a constant.

As Karlin and McGregor [5], we shall show that for each sufficiently small $\rho>0$,

$$
\left|1-e^{-x \hat{\theta}(w)}\right| \leq \frac{M e^{\rho x}}{|w|}, \quad \operatorname{Re} w \geq t_{0}
$$

To make the situation clear, we shall divide it into two cases.

Lemma 4. 1. If (4.1) holds with $1 / 2<p \leq 1$, then (4.3) holds.

The proof is the same as in [5] Lemma 5. But we shall prove for completeness. 
Proof. Choose an $\varepsilon$ satisfying $\pi(1-p) / 2 p<\varepsilon<\pi / 2$, and set

$$
D(\rho, \varepsilon)=\left\{\lambda ; 0<|\lambda|<\rho,|\arg \lambda|<\frac{\pi}{2}+\varepsilon\right\} .
$$

Then it is enough to show that for each sufficiently small $\rho$, a) $\left|\hat{\pi}(\lambda)\left(1-e^{-\lambda x}\right)\right| \leq \mathrm{Me}^{\rho x}$ for all $\lambda \in D(\rho, \varepsilon)$, b) $\hat{\pi}(\lambda)$ is univalent on $D(\rho, \varepsilon)$, c) $\hat{\pi}(\lambda)$ maps $D(\rho, \varepsilon)$ onto a domain containing a right halfplane $\operatorname{Re} w \geq t_{0}$.

Assertion a) follows immediately from (4.2). The substituation $z=1 / \lambda^{p}$ maps $D(\rho, \varepsilon)$ univalently onto

$$
D_{1}(\rho, \varepsilon)=\left\{z ;|z|>\frac{1}{\rho^{p}},|\arg z|<p\left(\frac{\pi}{2}+\varepsilon\right)\right\} .
$$

It is therefore sufficient to show that

$$
\psi(z)= \begin{cases}z+\frac{\beta}{\alpha} \log z+g_{3}\left(\frac{1}{z}\right), & \text { if } p=1, \\ z+c+z^{1-q} g_{4}\left(\frac{1}{z^{q}}\right), & \text { if } \frac{1}{2}<p<1,\end{cases}
$$

where $q=1 / p$, is univalent in $D_{1}(\rho, \varepsilon)$ and maps it onto a domain containing a right half-plane.

From (4.4) it follows that

$$
\left|\psi^{\prime}(z)-1\right|<L \rho, \quad z \in D_{1}(\rho, \varepsilon)
$$

for some $L>0$. Suppose that $z_{1}, z_{2}$ are distinct points of $D_{1}(\rho, \varepsilon)$ such that $\psi\left(z_{1}\right)=\psi\left(z_{2}\right)$. Then

$$
0=\int_{z_{1}}^{z_{2}} \psi^{\prime}(\zeta) d \zeta=\left(z_{2}-z_{1}\right)+\int_{z_{1}}^{z_{2}}\left(\psi^{\prime}(\zeta)-1\right) d \zeta
$$

From the geometry of $D_{1}(\rho, \varepsilon)$, it is clear that there is a constant $K$, depending on $\varepsilon$ but not on $\rho$, such that for any distinct points $z_{1}, z_{2}$ of $D_{1}(\rho, \varepsilon)$ there is a path $\Gamma$ lying in $D_{1}(\rho, \varepsilon)$ and jointing $z_{1}, z_{2}$ such that

$$
\text { length } \Gamma<K\left|z_{2}-z_{1}\right| \text {. }
$$

With this choice of the path of integration we have

$$
\left|\int_{z_{1}}^{z_{2}}\left(\psi^{\prime}(\zeta)-1\right) d \zeta\right|<L \rho K \mid z_{2}-z_{1} !
$$


and hence if $\rho$ is sufficiently small (4.5) is impossible, so $\psi(z)$ is univalent in $D_{1}(\rho, \varepsilon)$.

The mapping $w=\psi(z)$ sends the boundary of $D_{1}(\rho, \varepsilon)$ onto a continuous curve in the $w$-plane. When $z \rightarrow \infty$ on the ray $\arg z=$ $(\pi / 2+\varepsilon)$, inspection of (4.4) shows that $\operatorname{Re} w \rightarrow-\infty, \operatorname{Im} w \rightarrow+\infty$, since $p(\pi / 2+\varepsilon)>\pi / 2$. Similarly when $z \rightarrow \infty$ along the ray $\arg z=$ $-p(\pi / 2+\varepsilon)$, we have $\operatorname{Re} w \rightarrow-\infty, \operatorname{Im} w \rightarrow-\infty$. It follows that the image of $D_{1}(\rho, \varepsilon)$ contains a right half-plane.

Lemma 4.2. If (4.1) holds with $0<p \leq 1 / 2$, then (4.3) holds for the analytically continued function $\hat{\theta}(w)$.

Proof. Let us use the notations in the previous proof. Also, let $R(\rho, \varepsilon)$ be the image of $D_{1}(\rho, \varepsilon)$ by the function $\psi(z) / \alpha p$ in (4.4). Then the previous proof shows

$$
\hat{\theta}(w)=\frac{1}{\psi^{-1}(\alpha p w)^{q}}, \quad w \in R(\rho, \varepsilon),
$$

and $\hat{\theta}(w)$ is regular on the domain, maps it onto $D(\rho, \varepsilon)$. In this case however $R(\rho, \varepsilon)$ does not contain a right half-plane. So we shall take a new $\varepsilon_{0}>0$ such that $p\left(\pi / 2+\varepsilon_{0}\right)>\pi / 2$. For this $\varepsilon_{0}$, the previous argument shows that $\psi(z) / \alpha p$ is univalent also in the domain $D_{1}\left(\rho, \varepsilon_{0}\right)$ and maps it onto a domain $R\left(\rho, \varepsilon_{0}\right)$ containing a right half-plane. Hence $\psi^{-1}(\alpha p w)$ is regular on $R\left(\rho, \varepsilon_{0}\right)$, and maps it onto $D_{1}\left(\rho, \varepsilon_{0}\right)$, so that $\hat{\theta}(w)$ in (4.6) is analytically continued on the domain $R\left(\rho, \varepsilon_{0}\right)$, and maps it onto $D\left(\rho, \varepsilon_{0}\right)(=\{\lambda ; 0<|\lambda|<\rho\})$. Let $\hat{\theta}(w)=r e^{i \theta}, 0<r<\rho,|\theta|<\pi / 2+\varepsilon_{0}$. Then, (4.4) with (4.6) shows that

$$
\alpha p w=\frac{e^{-i p \theta}}{r^{p}}+c+\frac{r}{r^{p}} e^{i(1-p) \theta} g_{4}\left(r^{p} e^{i p \theta}\right) .
$$

Hence, we have

$$
\begin{aligned}
& \sup _{\substack{w \in R\left(\rho, \varepsilon_{0}\right) \\
w}}\left|\left(1-e^{-x \hat{\theta}(w)}\right) w\right| \\
& \quad \leq \sup _{\substack{0<r<\rho \\
|\theta|<\pi / 2+\varepsilon_{0}}}\left|\left(1-e^{-x r e^{i \theta}}\right)\left(\frac{e^{-i p \theta}}{r^{p}}+c+\frac{r}{r^{p}} e^{i(1-p) \theta} g_{4}\left(r^{p} e^{i p \theta}\right)\right)\right| / \alpha p \\
& \leq M e^{\rho x} .
\end{aligned}
$$

Theorem 4.1. Let (4.1) be satisfied. Then the transition function $P_{t}(x, E)$ admits a spectral representation 


$$
\begin{gathered}
P_{t}(x, E)=1-\int_{0}^{\infty} \beta_{u}(x) e^{-u\left(t-t_{0}\right)} \xi_{u}(E) d u, \quad t>t_{0}, \\
x \in[0, \infty), E \in \mathcal{K}[0, \infty),
\end{gathered}
$$

where $\beta_{u}(x)$ is in $L^{2}(0, \infty)$ as a function in $u$. Moreover if $p=1, \beta_{u}(x)$ is an eigenfunction of $P_{t}(x, E)$ when $u$ is a continuity point of $\beta_{u}(x)$.

Proof. From (4.3), it follows that the integrals

$$
\int_{-\infty}^{\infty}\left|1-e^{-x \hat{\theta}(r+i s)}\right|^{2} d \zeta, \quad r \geq t_{0}
$$

are uniformly bounded. Hence by appealing to the Paley-Wiener theorem ([6] p. 131) we deduce that

$$
1-e^{-x \hat{\theta}(w)}=\int_{0}^{\infty} e^{-w u} e^{t_{0} u} \beta_{u}(x) d u, \quad \operatorname{Re} w \geq t_{0},
$$

where $\beta_{u}(x)$ is in $L^{2}(0, \infty)$ as a function in $u$. Hence (2.6) in Condition A holds with

$$
\phi_{d u}(x)=\delta_{d u}(0)-e^{t_{0} u} \beta_{u}(x) d u .
$$

Take an $r>t_{0}$. Then Schwartz's inequality shows that

$$
\begin{aligned}
\int_{0}^{\infty} e^{-r u}|\phi|_{d u}(x) & \leq 1+\int_{0}^{\infty} e^{-\left(r-t_{0}\right) u}\left|\beta_{u}(x)\right| d u \\
& \leq 1+\left(\int_{0}^{\infty} e^{-2\left(r-t_{0}\right) u} d u\right)^{1 / 2}\left(\int_{0}^{\infty}\left|\beta_{u}(x)\right|^{2} d u\right)^{1 / 2}<\infty .
\end{aligned}
$$

Hence (2. 7) follows, and Lemma 2.4 implies (4.7) since $\xi_{0}(E)=\delta_{E}(0)$ by (2.1).

To show the latter assertion, note that (4.8) is written as

$$
1-e^{-x \hat{\theta}(r+2 \pi i s)}=\int_{0}^{\infty} e^{-2 \pi i s} e^{-\left(r-t_{0}\right) u} \beta_{u}(x) d u, \quad r>t_{0},
$$

and hence by Parseval's equality and (4.3)

$$
\int_{0}^{\infty} e^{-2\left(r-t_{0}\right) u}\left|\beta_{u}(x)\right|^{2} d u \leq \frac{M^{2}}{2\left(r-t_{0}\right)} e^{2 \rho x}, \quad r>t_{0} .
$$

So for $w>r>t_{0}$, Schwartz's inequality with (4.9) shows

$$
\begin{aligned}
\int_{0}^{\infty} e^{-w u}|\phi|_{d u}(x) & \leq 1+\int_{0}^{\infty} e^{-w u}\left|\beta_{u}(x)\right| d u \\
& \leq 1+\left(\int_{0}^{\infty} e^{-2(w-r) u} d u\right)^{1 / 2}\left(\int_{0}^{\infty} e^{-2\left(r-t_{0}\right) u}\left|\beta_{u}(x)\right|^{2} d u\right) \\
& \leq M^{\prime} e^{\rho x}
\end{aligned}
$$


where $M^{\prime}$ is a constant independent of $x$. Since $h(\lambda)$ is analytic at 0 by the assumption, we have

$$
\int_{0}^{\infty} P_{t}(x, d y) e^{\rho y}<\infty
$$

for sufficiently small $\rho>0$. (4.10) and (4.11) imply (2.8) in Condition B. Hence by Lemma 2.3, we see that $\phi_{d u}(x)$ is an eigenfunction of $P_{t}(x, E)$. The desired assertion follows immediately from (2.9) and (4.9).

Remark 4. 1. If $g(\lambda)$ in (4.1) is a constant, then $t_{0}$ in (4.7) may be taken as 0 . Indeed, in this case, the radius $\rho$ in Lemmas 4.1 and 4.2 can be taken as $\infty$, and if $1 / 2<p \leq 1, \psi(z)$ maps $D_{1}(\infty, \varepsilon)$ onto a domain containing the right half-plane $\operatorname{Re} w>0$, and if $0<p \leq 1 / 2, \hat{\theta}(w)$ can be analytically continued to a domain containing the right half-plane. Hence by the same arguments as in Lemmas 4. 1 and 4.2, we have (4.3) for $\operatorname{Re} w>0$, obtaining (4.7) for $t>0$ (cf. Example 3 given below).

Proposition 4.1. If (4.1) holds, then

(4. 12) $\quad \psi_{t}(\lambda)= \begin{cases}\frac{1}{\alpha t}+\frac{\beta \log t}{\alpha^{3} t^{2}}-\frac{\hat{\pi}(\lambda)+c_{1}}{\alpha^{2} t^{2}}+0\left(\frac{(\log t)^{2}}{t^{3}}\right), & p=1, \\ \frac{1}{(\alpha p t)^{q}}-\frac{\alpha \hat{\pi}(\lambda)-q c}{(\alpha p t)^{q+1}}+0\left(\frac{1}{t^{q+2}} \vee \frac{1}{t^{2 q}}\right), & 0<p<1,\end{cases}$

as $t \rightarrow \infty$, where 0() are uniform in $\lambda \geq K$ for each $K>0$, and $\alpha, \beta, q, c$ are constants given in the proof of Lemma 4.1 and $c_{1}=-\left(\beta \log \alpha+g_{3}(0)\right) / \alpha^{2}$. In words of semigroups, (4.12) means

$$
\text { (4.13) } E_{x}\left(e^{-\lambda x t}\right)=\left\{\begin{array}{c}
1-x\left(\frac{1}{\alpha t}+\frac{\beta \log t}{\alpha^{3} t^{2}}-\frac{\hat{\pi}(\lambda)+c_{1}}{\alpha t^{2}}\right)+\frac{x^{2}}{2 \alpha^{2} t^{2}}+0\left(\frac{(\log t)^{2}}{t^{3}}\right), \\
1-x\left(\frac{1}{(\alpha p t)^{q}}-\frac{\alpha \hat{\pi}(\lambda)-q c}{(\alpha p t)^{q+1}}\right)+0\left(\frac{1}{t^{q+2}} \vee \frac{1}{t^{2 q}}\right), \\
0<p<1
\end{array}\right.
$$

as $t \rightarrow \infty$. The asymptotic behavior of the probability $P_{x}\left(\sigma_{\{0\}} \leq t\right)$ as $t \rightarrow \infty$ is given also by (4.13) with $\hat{\pi}(\lambda)$ replaced by 0 .

Proof. Let $p=1$ first. Then by virtue of (4.2), 
(4. 14) $\hat{\theta}(w)=\frac{1}{\alpha w}\left(1-\hat{\theta}(w) \log \hat{\theta}(w)+\hat{\theta}(w) g_{3}(\hat{\theta}(w)), \quad w \rightarrow \infty\right.$

Since $\hat{\theta}(w) \rightarrow 0(w \rightarrow \infty),(4.14)$ implies

$$
\hat{\theta}(w)=\frac{1}{\alpha w}+o\left(\frac{1}{w}\right), \quad w \rightarrow \infty,
$$

which with (4.14) shows

$$
\hat{\theta}(w)=\frac{1}{\alpha w}+0\left(\frac{\log w}{w^{2}}\right), \quad w \rightarrow \infty .
$$

Substituting (4.15) into (4.14), we have

$$
\hat{\theta}(w)=\frac{1}{\alpha w}\left(1+\frac{\beta \log }{\alpha^{2} w}-\frac{c_{1}}{w}+0\left(\frac{(\log w)^{2}}{w^{2}}\right)\right), \quad w \rightarrow \infty,
$$

and hence, by (1.10) we obtain (4.12) for $p=1$.

When $0<p<1$, (4.2) implies

$\hat{\theta}(w)^{p}=\frac{1}{\alpha p w}\left(1+\frac{c}{\alpha p w}+0\left(\frac{1}{w^{2}}\right)\right)+\frac{\hat{\theta}(w) g(\hat{\theta}(w))}{\alpha p w}\left(1+0\left(\frac{1}{w}\right)\right), \quad w \rightarrow \infty$, and the similar arguments show (4.12) for $0<p<1$. The rest assertions are easily obtained as in the proof of Proposition 3.1.

Remark 4.2. The higher approximations may be obtained by the same methods.

Remark 4.3. If we assume

$$
h(\lambda)=-\alpha \lambda^{1+p}(1+o(1)), \quad \lambda \downarrow 0,
$$

instead of (4.1), we have

$$
\psi_{t}(\lambda)=\frac{1}{(\alpha p t)^{q}}+o\left(\frac{1}{t^{q}}\right), \quad t \rightarrow \infty .
$$

\section{$\S 5$. Examples of the Representation}

Example 1. $h(\lambda)=-a \lambda^{1+p}+b \lambda, a, b>0,0<p \leq 1 . \quad h(\lambda)$ is supercritical and satisfies (1.5). The positive solution $\gamma$ of $h(\lambda)=0$ is $(b / a)^{q}$, where $q=1 / p . \quad \hat{\pi}(\lambda)$ in $(1.8)$ is

$$
\hat{\pi}(\lambda)=-\frac{1}{p b} \log \left(1-\frac{b}{a \lambda^{p}}\right), \quad \lambda>\gamma,
$$


and the stationary measure is

$$
\pi(d x)=\frac{1}{p b} \sum_{l=1}^{\infty}\left(\frac{b}{a}\right)^{l} \frac{x^{p l-1}}{l \Gamma(p l)} d x .
$$

Since $\mu=-h^{\prime}(\gamma)=p b, A(\lambda)$ in Lemma 3.1 is

$$
A(\lambda)=1-\frac{b}{a \lambda^{p}}, \quad \lambda>0 .
$$

Hence,

$$
\xi_{k \mu}(d x)=\sum_{l=1}^{k}\left(\begin{array}{c}
k \\
l
\end{array}\right)\left(-\frac{b}{a}\right)^{l} \frac{x^{p l-1}}{\Gamma(p l)} d x+\delta_{d x}(0) .
$$

The inverse function $B(v)$ of $A(\lambda)$ is

$$
B(v)=\gamma\left(1+\frac{v}{1-v}\right)^{q}, \quad|v|<1 .
$$

Hence, if we let

$$
P_{\nu, k}(x)= \begin{cases}\sum_{l=1}^{[k / \nu]} \frac{1}{l !}\left(\begin{array}{c}
k-1 \\
\nu l-1
\end{array}\right)\left[-\gamma x\left(\begin{array}{l}
q \\
\nu
\end{array}\right)\right]^{l}, & k \geq \nu, \\
1, & k=0,\end{cases}
$$

$\phi_{k \mu}(x)$ is given by

$$
\phi_{k \mu}(x)=e^{-\gamma x} \sum_{\substack{k_{1}+\ldots+k_{k}=k \\ k_{\nu} \geq \nu \text { or } k_{\nu}=0}} \prod_{\nu=1}^{k} P_{\nu, k_{\nu}}(x) .
$$

When $p=1$, the corresponding C.B.P. is a diffusion corresponding to the backward equation

$$
\frac{\partial u}{\partial t}=a x \frac{\partial^{2} u}{\partial x^{2}}+b x \frac{\partial u}{\partial x}
$$

(cf. [7]), and $\xi_{k \mu}(d x), \phi_{k \mu}(x)$ in (5.1) and (5.3) are

$$
\begin{aligned}
& \xi_{k \mu}(d x)= \begin{cases}\left(-\frac{b}{a} x\right) L_{k-1}^{(1)}(b x / a) d x / x+\delta_{d x}(0), & k \geq 1, \\
\delta_{d x}(0), & k=0,\end{cases} \\
& \phi_{k \mu}(x)= \begin{cases}\left(-\frac{b}{a} x\right) L_{k-1}^{(1)}(b x / a) e^{-\gamma x}, & k \geq 1, \\
e^{-\gamma x}, & k=0,\end{cases}
\end{aligned}
$$

where $L_{k}^{(\alpha)}(x)$ is the Laguerre's polynomial. Hence 


$$
P_{t}(x, E \backslash\{0\})=\sum_{k=1}^{\infty} \phi_{k}^{0}(x) e^{-k \mu t} \int_{E \backslash\{0\}} \phi_{k}^{0}(y) m(d y), \quad t>0,
$$

where $\phi_{k}^{0}(x)=\left(-b x e^{-\gamma x} / a\right) L_{k-1}^{(1)}(b x / a)$ and $m(d x)=e^{\gamma x} d x / x . \quad m(d x)$ is the canonical measure of the diffusion process.

Example 2. $h(\lambda)=a\left(\lambda+(b / a)^{q}\right)^{1+p}+b\left(\lambda+(b / a)^{q}\right), a, b>0,0<p \leq 1$ and $q=1 / p$. In this case $h(0)=0, h^{\prime}(0)=-p b<0$. Hence, $h(\lambda)$ is subcritical. $h(\lambda)$ satisfies (1.5), and the smaller solution $-\gamma_{0}$ of $h(\lambda)=0$ is $-(b / a)^{q}$. Moreover,

$$
\hat{\pi}(\lambda)=-\frac{1}{p b} \log \left(1-\frac{b}{a\left(\lambda+\gamma_{0}\right)^{p}}\right),
$$

and the stationary measure is

$$
\pi(d x)=\frac{1}{p b} \sum_{l=1}^{\infty}\left(\frac{b}{a}\right)^{l} \frac{x^{p l^{-1}}}{l \Gamma(p l)} e^{-\gamma_{0} x} d x .
$$

$\xi_{k \mu}(d x)$ and $\phi_{k \mu}(x)$ are

$$
\begin{aligned}
& \xi_{k \mu}(d x)=\sum_{l=1}^{k}\left(\begin{array}{c}
k \\
l
\end{array}\right)\left(-\frac{b}{a}\right)^{l} \frac{x^{p l-1}}{\Gamma(p l)} e^{-\gamma_{0} x} d x+\delta_{d x}(0), \\
& \phi_{k \mu}(x)=\sum_{\substack{k_{1}+\ldots+k_{k}=k \\
k_{\nu} \geq \nu \text { or } k_{\nu}=0}} \prod_{\nu=1}^{k} P_{\nu, k_{\nu}}(x),
\end{aligned}
$$

where $P_{v, k}(x)$ is that of (5.2).

When $p=1$, the C.B.P. is a diffusion with the backward equation

$$
\frac{\partial u}{\partial t}=a x \frac{\partial^{2} u}{\partial x^{2}}-b x \frac{\partial u}{\partial x}
$$

and the transition function admits the spectral representation

$$
P_{t}(x, E \backslash\{0\})=\sum_{k=1}^{\infty} \phi_{k}^{0}(x) e^{-k \mu t} \int_{E \backslash\{0\}} \phi_{k}^{0}(y) m(d y), \quad t>0,
$$

where $\phi_{k}^{0}(x)=(-b x / a) L_{k-1}^{(1)}(b x / a)$ and $m(d x)=e^{-\gamma_{0} x} d x / x . \quad m(d x)$ is the canonical measure for the diffusion process.

Example 3. $h(\lambda)=-a \lambda^{1+p}, a>0,0<p \leq 1$. This is the simplest case of (4.1).

$$
\hat{\pi}(\lambda)=\frac{1}{p a \lambda^{p}}, \quad \lambda>0,
$$




$$
\begin{aligned}
& \pi(d x)=\frac{x^{p-1}}{p a \Gamma(p)} d x, \\
& \xi_{u}(d x)=\sum_{l=1}^{\infty} \frac{1}{l !}\left(-\frac{u}{p a}\right)^{l} \frac{x^{p l-1}}{\Gamma(p l)} d x+\delta_{d x}(0), \\
& \phi_{d u}(x)=\sum_{l=1}^{\infty} \frac{1}{l !} \frac{(-x)^{l}}{(p a)^{q l}} \frac{u^{q l-1}}{\Gamma(q l)} d u+\delta_{d u}(0) .
\end{aligned}
$$

When $p=1$, the C.B.P. is a diffusion process with the backward equation

$$
\frac{\partial u}{\partial t}=a x \frac{\partial^{2} u}{\partial x^{2}}
$$

and the transition function has the spectral representation

$$
P_{t}(x, E \backslash\{0\})=\int_{0}^{\infty} \int_{E \backslash\{0\}} \phi_{u}^{0}(x) e^{-t u} \phi_{u}^{0}(y) m(d y) d u,
$$

where $\phi_{u}^{0}(x)=\sqrt{x / a} J_{1}(\sqrt{4 u x / a})^{4)}$ and $m(d x)=d x / x . \quad m(d x)$ is the canonical measure for the diffusion.

\section{References}

[1] Feller, W., An Introduction to Probability and its Applications, II, Wiley, New York, 1966.

[2] Harris, T. E., The Theory of Branching Processes, Springer, Berlin-GöttingenHeiderberg, 1963.

[3] Jirina, M., Stochastic branching process with continuous state space, Czechoslovak Math. J. 8 (1958), 292-313.

[4] Karlin, S. and J. McGregor, Spectral theory of branching processes, I, II, Z. Wahrsheinlichkeitstheorie und Verw. Gebiete 5 (1966), 1-33; 34-54.

[5] - On the spectral representation of branching processes with mean one, J. Math. Anal. Appl. 21 (1968), 485-495.

[6] Hoffman, K., Banach Spaces of Analytic Functions, Prentice-Hall, New Jersey, 1962.

[7] Lamperti, J., Continuous state branching processes, Bull. Amer. Math. Soc. 73 (1967), 381-386.

[8] Watanabe, S., On two dimensional Markov processes with branching property, Trans. Amer. Math. Soc. 36 (1969), 447-466.

[9] Widder, D. V., The Laplace Transform, Princeton University Press, 1946.

4) $J_{1}(x)$ is the Bessel's function. 
